\title{
Flow Kinetics in Porous Ceramics: Understanding with Non-Uniform Capillary Models
}

\author{
Debdutt Patro, Saswata Bhattacharyya, and Vikram Jayaram ${ }^{*, \dagger}$ \\ Department of Materials Engineering, Indian Institute of Science, Bangalore -560012, India
}

\begin{abstract}
The present work describes the development of a two-parameter non-uniform capillary model to describe kinetics of flow in porous solids with complex tortuous varying paths. Experimentally, the rate of fluid flow in such a non-uniform capillary is found to be orders of magnitude slower compared with a corresponding average uniform capillary. This slow rate is explained in terms of an extremely small 'effective' hydrodynamic radius. The origin of such an 'unphysical' radius is rationalized based on geometrical considerations and effective driving forces for flow through a stepped capillary. Infiltration rate parameters are derived from the geometry of the porous medium for both wetting and non-wetting conditions.
\end{abstract}

\section{Introduction}

$\mathrm{F}$ LOw of fluids through porous media is frequently encountered in a large number of processes encompassing diverse disciplines, e.g., processing of composites by melt infiltration, forced oil recovery, and water flow through soils. The efficiency of such processes is to a large extent governed by the rate of fluid flow through the medium, wherein the infiltrating liquid is either a part of the system (composite processing via the directed metal oxidation (DIMOX), ${ }^{1}$ pressureless melt infiltration (PRIMEX) ${ }^{2}$ and reaction-bonded $\mathrm{SiC}$ (REFEL) ${ }^{3}$ techniques), or used for material transport (as in biological/geological processes). In particular, melt infiltration in composite processing is a complex process based on an interplay of physical phenomena (i.e., fluid flow through porous solid) and chemical effects (related to wettability of reinforcement, and formation of interfacial reaction products that dictate capillary-induced melt flow). Composite formation rates, e.g., $\left(\mathrm{Al} / \mathrm{Al}_{2} \mathrm{O}_{3}\right.$, glass $\left./ \mathrm{Al}_{2} \mathrm{O}_{3}, \mathrm{Si} / \mathrm{SiC}, \mathrm{CoSi}{ }_{2} / \mathrm{SiC}\right)$ are found to be extremely slow ${ }^{4-7}$ in the range of $\mathrm{mm} / \mathrm{h}-\mathrm{cm} / \mathrm{h}$. Such rates are two to three orders of magnitude slower than that predicted by viscous flow-controlled rise through a 'pore size distribution-averaged' uniform capillary, which predicts infiltration velocities of the order $\sim \mathrm{mm} / \mathrm{sec}$. Reported activation energies $^{8,9}$ are in the range of few $100 \mathrm{~kJ} / \mathrm{mol}$, indicating a chemical reaction-controlled kinetics.

The influence of alloying additions on kinetics has been extensively studied in sessile drop spreading experiments wherein extremely slow drop-spreading rates (four to five orders of magnitude slower than that dictated by viscous hydrodynamic flow) have been attributed to (a) diffusion-limited spreading ${ }^{10}$ and (b) local reaction-controlled spreading. ${ }^{11}$ Similar effects during infiltration into porous bodies have been reported: $\mathrm{Al} / \mathrm{TiC}^{12}$ and $\mathrm{NiSi} / \mathrm{C}^{13}$ Capillary flow in such reactive couples is governed by bulk diffusion of the solute as well as the local reaction rates of

L. Klein - contributing editor

Manuscript No. 21962. Received June 30, 2006; approved April 9, 2007.

Financial support for the work was provided by a grant from Volkswagen Foundation.

${ }^{*}$ Member, American Ceramic Society

Author to whom correspondence should be addressed. e-mail: qjayaram@materials. iisc.ernet.in the moving melt meniscus with the ceramic reinforcement, both of which ensure good wettability.

Over and above such complexities is the issue of contact angle-driven melt flow through the porous medium, which could possibly be rate limiting for non-reactive systems.

Based on the topology of the porous medium, fluid flow has been analyzed as (a) structural models, which describe the porous media with a particular geometry and (b) non-structural models based on hydraulic radius theory, which consider average properties, i.e., porosity and specific surface area of porous medium (e.g., Carman-Kozeny equation ${ }^{14}$ ). We curtail our discussion for non-structural models and restrict ourselves to structural models for the current study.

Structural models provide ease of visualization of fluid flow in porous media. Washburn ${ }^{15}$ first used a capillary bundle model ${ }^{16}$ to describe the structure of a porous body by considering the porous medium as a bundle of uniform capillaries with an 'effective' radius $r_{\text {eff. }}$. Kinetics of flow in the early stages can be given by the Washburn equation

$$
\begin{aligned}
& h^{2}=\frac{r_{\mathrm{eff}} \gamma \cos \theta}{2 \eta} t \\
& v=\frac{r_{\mathrm{eff}} \gamma \cos \theta}{4 \eta h}
\end{aligned}
$$

where $\gamma=$ liquid surface tension, $\theta=$ contact angle, $\eta=$ liquid viscosity, and $v=$ meniscus velocity.

However, the drawback with such a model is that an equivalent capillary radius cannot be ascribed to the porous media due to constrictions and expansions present in flow paths in real porous solids. Typically, kinetics of capillary rise by taking an average pore radius (based on the pore size distribution) was found to be two to three orders of magnitude faster than that observed experimentally. ${ }^{4}$

This anomaly necessitates a structural model, which incorporates the essential converging-diverging nature of flow path in a porous medium and can explain the slow flow rates observed. An attempt to solve this was reported by Dullien et al. ${ }^{17}$ where the nature of flow in porous media was approximated by a simple stepped tube repeating unit model. Such a model ascribed the slow rise in porous media to the extremely small driving forces on the meniscus in the diverging sections and concomitantly small velocities. Based on the two-segment repeating unit, Einset ${ }^{6}$ explained infiltration rates of silicon into porous carbon. Sinusoidal capillary ${ }^{18,19}$ models with converging-diverging sections have been developed as more realistic representation of flow paths in porous bodies. The effect of multidimensional flow in porous media has been addressed by incorporating a tortuosity factor of 3 (for random network structure comprised of one-dimensional (1D) non-uniform capillaries) and network theory. ${ }^{20,21}$ However, the underlying physics of flow in such nonuniform capillaries and effective forces acting on the moving liquid have not been adequately addressed. The current work is an attempt to develop a non-uniform capillary model based on measurable parameters of the porous medium in a model system and to generalize it to the different conditions used in the 
processing of composites, viz., melt infiltration under pressure as well as pressureless infiltration.

While the fluid flow model developed in the work cannot strictly be applied to reactive systems, we explore situations in which the present results may serve as an upper bound for the infiltration kinetics.

\section{Experimental Procedure}

Infiltration runs were conducted with polyethylene glycol (PEG 600, Merck, Mumbai, India) as the liquid and white fused $\mathrm{Al}_{2} \mathrm{O}_{3}$ (M/s Grindwell Norton, Mumbai, India) porous beds as well as slip-cast $\mathrm{Al}_{2} \mathrm{O}_{3}$ samples. Powder preparation and bed preparation were carried out meticulously as described subsequently for ensuring reproducibility of results.

\section{(1) Powder Preparation}

As-received powders were classified by sieving. Sieved powders were washed with water and heated to $1200^{\circ} \mathrm{C}$ in order to remove impurities.

\section{(2) Powder Bed Preparation}

A small quantity of powder was poured into a glass tube and tapped until constant bed height was achieved. This process was repeated until desired bed height of $8 \mathrm{~cm}$ was obtained. Porosity was calculated during bed preparation based on visual measurement of height and corresponding weight. Values obtained for the different beds studied are presented in Table I, column 2.

\section{(3) Slip-Cast Sample}

Methyl cellulose was used as a binder and 25-37 $\mu \mathrm{m}$ particles were slip-cast with a solid loading of $\sim 60 \%$. The larger sizes could not be cast due to faster settling. Angular particles used in the study do not pack very efficiently and thus a packing density in the slip-cast product, similar to the loose powder-packed bed, could be obtained. The slips were fired at $1400^{\circ} \mathrm{C}$ for handling strength.

\section{(4) Infiltration Setup}

A permeable cloth was firmly tied to the bottom open end of the tube to support the packed powder bed. The packed tube was suspended over an empty beaker from a weighing balance as shown (Fig. 1). PEG was slowly poured inside the beaker until the lower $10 \mathrm{~mm}$ of the tube was immersed under the liquid. Weight readings as a function of time were taken after $5 \mathrm{~min}$ of immersion to avoid transient effects. Simultaneously, interface positions were noted visually as a function of time. Infiltration was conducted into a wide variety of packed loose beds (Table I). A typical plot showing infiltration kinetics for a $25-37 \mu \mathrm{m}$ porous bed is shown in Fig. 2. Experiments were repeated for reproducibility. Infiltration profiles for different beds were fitted to parabolic functions (Washburn equation) to obtain the 'effective' capillary dimensions for the packed bed. Fitted values for different beds using fluid properties $\gamma, \eta, \rho,{ }^{22}$ and $\theta$ are shown in Table II, $\eta=18.3 \times 10^{-3}$ Pa.s, $\gamma=0.048 \mathrm{~J} / \mathrm{m}^{2}$, $\rho=1.13 \times 10^{3} \mathrm{~kg} / \mathrm{m}^{3}$, and $\theta=41^{\circ}$ (static contact angle of sessile

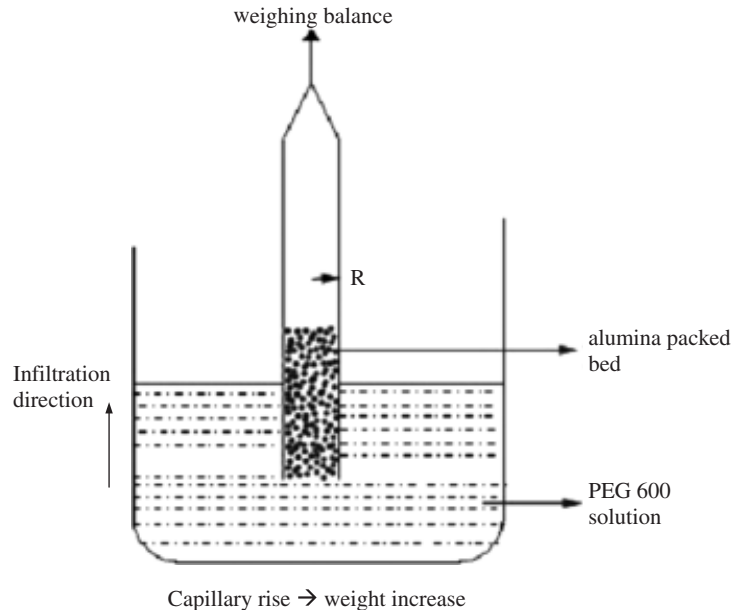

Fig. 1. Schematic of the setup for measuring infiltration kinetics.

drop on polished $\mathrm{Al}_{2} \mathrm{O}_{3}$ substrate measured using a goniometer in the present study).

It is possible that the particles may rearrange during infiltration, thereby changing the pore size distribution. However, no movement of the top of the bed could be seen during infiltration, possibly due to the limited rearrangement of the angular particles as a result of large inter-particle frictional forces.

Porous packed beds were characterized using $\mathrm{Hg}$ porosimetry (Poremaster PM 60GT, Quantachrome Instruments, Boynton Beach, FL). However, the bed disintegrated during pressurization. To circumvent this problem, sintered versions of the packed compacts having sufficient strength were characterized for pore size distribution. No crushing of the slip-cast compacts occurred on pressurization up to the maximum pressure of 5000 psi ( $\sim 35 \mathrm{MPa})$. The cumulative intrusion curve for the slip-cast and sintered $\left(1400^{\circ} \mathrm{C}\right)$ powder bed is shown in Fig. 3 .

\section{Results and Discussion}

The pore size distribution of $0.4-30 \mu \mathrm{m}$ (Fig. 3) for a slip-cast 25-37 $\mu \mathrm{m}$ sample was considered representative of pore size distribution for the 25-37 $\mu \mathrm{m}$ loose powder-packed bed based on the preparation methodology of the two samples, as shown later. The distribution was truncated at the lower and upper ends of the volumetric pore size distribution, $\mathrm{D}_{V}(\mathrm{~d})$, so as to exclude $5 \%$ pore volume, as the remaining $95 \%$ volume of pores would still control majority of the flow. This leads to a relevant pore size distribution of $1.4-10.8 \mu \mathrm{m}$ for the $25-37 \mu \mathrm{m}$ packed bed. For comparison, we have considered a $90 \%$ volumetric pore size distribution as well, which gives an effective pore size distribution of $2.2-10.3 \mu \mathrm{m}$. The results of infiltration kinetics for both cases will be presented subsequently.

Room-temperature imbibition of PEG 600 into different packed alumina beds provides us with a quantitative estimate of the influence of bed pore size distribution on flow kinetics. Precautions were taken to exclude the effect of other factors, viz., buoyancy, surface tension, etc., on weight gain readings.

Table I. 'Effective Radii' for the Different Beds Obtained from Parabolic $h^{2}-t$ Fits

\begin{tabular}{|c|c|c|c|c|}
\hline & Packed bed type & Porosity $(\%)$ & $\begin{array}{c}\text { 'Effective' } \\
\text { Washburn radius }(\mu \mathrm{m}) \\
\text { (weight-time readings) }\end{array}$ & $\begin{array}{c}\text { 'Effective' Washburn } \\
\text { radius }(\mu \mathrm{m}) \\
\text { (height-time readings) }\end{array}$ \\
\hline 1 & $25-37 \mu \mathrm{m}$ angular $\mathrm{Al}_{2} \mathrm{O}_{3}$ & 52 & $0.06 \pm 0.02$ & - \\
\hline 2 & $25-37 \mu \mathrm{m}$ spherical $\mathrm{Al}_{2} \mathrm{O}_{3}$ & 40 & $0.037 \pm 0.01$ & 0.047 \\
\hline 3 & $63-75 \mu \mathrm{m}$ angular $\mathrm{Al}_{2} \mathrm{O}_{3}$ & 49 & $0.25 \pm 0.01$ & 0.38 \\
\hline 4 & $63-75 \mu \mathrm{m}$ spherical $\mathrm{Al}_{2} \mathrm{O}_{3}$ & 42 & $0.1 \pm 0.01$ & 0.09 \\
\hline 5 & 25-37 $\mu \mathrm{m}$ angular $\mathrm{Al}_{2} \mathrm{O}_{3}$ slip-cast and sintered compact & 51 & $0.0 \overline{7} 7$ & - \\
\hline
\end{tabular}




\section{PEG 600 infiltrating $25-37 \mu \mathrm{m}$ angular $\mathrm{Al}_{2} \mathrm{O}_{2}$ compacts}

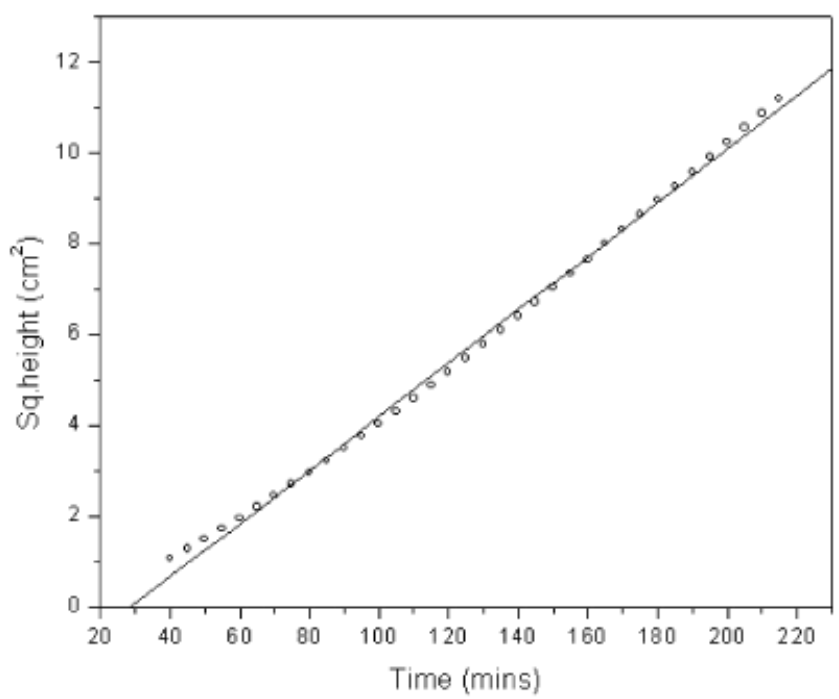

Fig. 2. Measured capillary rise kinetics of polyethylene glycol (PEG) 600 inside porous $25-37 \mu \mathrm{m} \mathrm{Al}_{2} \mathrm{O}_{3}$ compacts and the corresponding parabolic fit.

The weight gain during infiltration was converted into an equivalent height according to the relation

$$
\frac{\mathrm{d} w}{\mathrm{~d} t}=\rho\left(\frac{\mathrm{d} V}{\mathrm{~d} t}\right)=\rho \pi R^{2} \varepsilon\left(\frac{\mathrm{d} h}{\mathrm{~d} t}\right)
$$

where $\mathrm{d} w / \mathrm{d} t=$ weight increase rate, $\mathrm{d} h / \mathrm{d} t=$ height increase rate, $\rho=$ density of PEG $600, R=$ internal radius of glass tube used for making bed, and $\varepsilon=$ bed porosity.

Height-time data (derived from weight-time data as well as from interface tracking) for various compacts fit a parabolic 'Washburn' (Eq. (1a)).

A representative plot of $h^{2}$ versus $t$ is shown in Fig. 2. The infiltration rate parameter was determined by linear fit to the data points.

A good parabolic fit implies a laminar, steady-state flow on a macroscopic scale inside the porous bed. It establishes equivalence between flow kinetics through the porous media and a bundle of uniform capillaries with radii given by $r_{\text {eff }}$ as per Eq. (1) and previously pointed out by other researchers. ${ }^{16}$ The 'effective' radii for different porous beds are presented in Table I. Comparing different particle sizes (Table I), we notice that beds with larger particle sizes exhibit faster infiltration kinetics compared with smaller particle sizes due to their larger average pore sizes. Also, effective radii calculated from both weight-time and height-time readings are in good agreement. Effective radius, $r_{\text {eff }}$, of the slip-cast $25-37 \mu \mathrm{m}$ compact is similar to the 25-37 $\mu \mathrm{m}$ loose powder $\mathrm{Al}_{2} \mathrm{O}_{3}$ compact (Table I), and hence it is reasonable to assume that these have similar pore size distributions and indicates that there is not much particle rearrangement during infiltration.

The Washburn radii, $r_{\text {eff }}$, obtained from $\mathrm{d} h / \mathrm{d} t$ readings are in general larger than $r_{\text {eff }}$ obtained from $\mathrm{d} w / \mathrm{d} t$ readings, suggesting

Table II. Effective 'Hydrodynamic' Radii for Pressureless Infiltration of PEG 600 into $\mathrm{Al}_{2} \mathrm{O}_{3}$ Stepped Capillaries

\begin{tabular}{llllll}
\hline $\begin{array}{l}r_{\max } \\
(\mu \mathrm{m})\end{array}$ & $\begin{array}{c}r_{\min } \\
(\mu \mathrm{m})\end{array}$ & $\begin{array}{c}r_{\text {avg }} \\
(\mu \mathrm{m})\end{array}$ & $\begin{array}{c}r_{\text {avg, } V / S} \\
(\mu \mathrm{m})\end{array}$ & $\begin{array}{c}r_{\text {eff }}=\frac{r_{\min }^{4}}{r_{\max }^{m}} \\
(\text { Eq. }(12))(\mu \mathrm{m})\end{array}$ & $\begin{array}{c}r_{\text {eff }} \text { (Eq. (7)) } \\
(\text { modeled }) \mu \mathrm{m}\end{array}$ \\
\hline $0.7^{\dagger}$ & 0.7 & 0.7 & 0.7 & 0.7 & 0.7 \\
1.4 & 0.7 & 1.05 & 1.17 & 0.09 & 0.29 \\
$5.4^{\ddagger}$ & 0.7 & 3.05 & 4.86 & 0.002 & 0.007 \\
10.5 & 0.7 & 5.6 & 9.89 & 0.0002 & 0.0009 \\
\hline
\end{tabular}

${ }^{\dagger}$ Uniform capillary. ${ }^{\ddagger}$ Pore size distribution for $25-37 \mu \mathrm{m}$ packed bed.

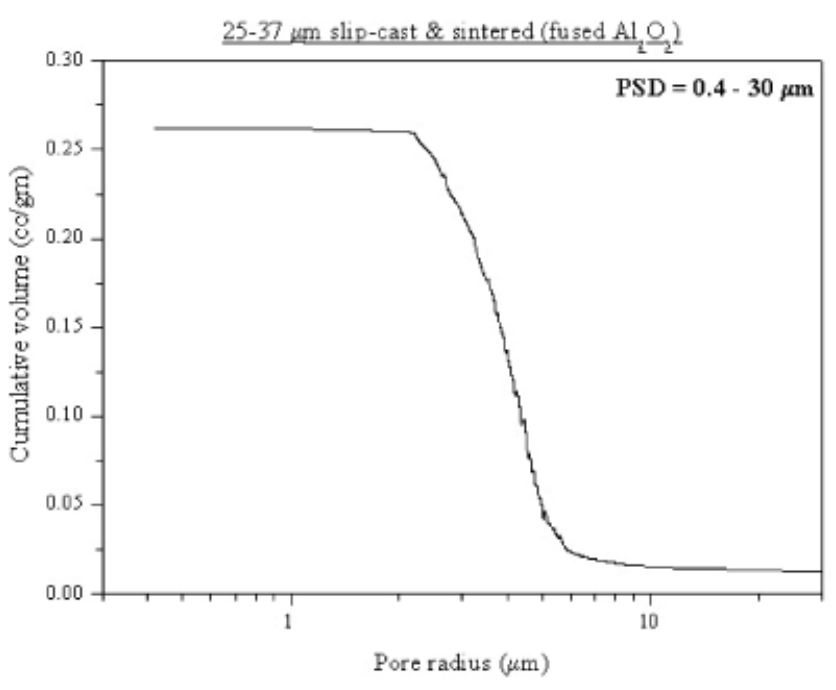

Fig. 3. Cumulative volumetric pore distribution curve from $\mathrm{Hg}$ intrusion porosimetry with a pore radius distribution of $0.2-15 \mu \mathrm{m}$.

a redundant pore volume of $10-15 \%$. This can be usually expected for a spontaneous rise, wherein $10-20 \%$ of the pores are left filled with air. However, this does not significantly influence the values of "hydrodynamic" radii obtained by parabolic fits to the profile.

For the 25-37 $\mu \mathrm{m}$ angular bed, we find $r_{\text {eff }}$ to be $0.06 \mu \mathrm{m}$ using a static contact angle of $41^{\circ}$. It is known that static contact angles may not reflect the actual dynamic contact angle inside the porous medium and the advancing meniscus typically has a larger contact angle that is dependent on the meniscus velocity. ${ }^{23}$ For the present case, this turns out to be small enough $\left(\mathrm{Ca}=10^{-5}\right.$, where $\mathrm{Ca}=$ capillary number $)$ not to distort the contact angle. In a different approach, ${ }^{24}$ both the 'effective' radius as well as the contact angle were considered to be variables for infiltration inside a porous medium, which were extracted by fitting the saturation profile to the Lucas-Washburn equation. Typically, the 'effective' contact angle was found to be larger than the static contact angle ${ }^{25}$ and the corresponding 'effective' hydrodynamic radius was found to be larger than that calculated from the static contact angle, due to inclusion of the gravitational potential term. However, the underlying equivalence between the rise in porous media and rise in uniform capillaries is based on the fact that the static contact angles can be used for the uniform capillary, the only variable being the effective Washburn radius for infiltration heights where gravity can be neglected, as in the present study. The importance of 'effective' local contact angle for the kinetics of infiltration will be highlighted later in the section on the sinusoidal capillary model.

The striking finding is that 'effective radii' in general for all beds are at least one to two orders of magnitude smaller than the average particle sizes. A better comparison is made between the 'effective hydrodynamic' radius, $r_{\text {eff }}$ (Table I), and the average pore size obtained from the pore size distribution. The average pore size was extracted from the pore size distribution in two ways: (a) arithmetic mean pore size, i.e., $r_{\text {avg }}=1 / 2\left(r_{\min }+r_{\max }\right)$ and (b) volume/surface area ratio based, i.e., $r_{\mathrm{avg}, V / S}$ $=\left(r_{\min }+r_{\max }\right)-\left(\frac{2 r_{\min } r_{\max }}{r_{\min }+r_{\max }}\right)$. The second expression can be considered to be analogous to an 'effective hydraulic' radius. Typically, $r_{\mathrm{avg}, V / S}$ was found to be greater than $r_{\mathrm{avg}}$. A comparison between the two different representations, viz., $r_{\text {avg }}$ and $r_{\text {avg, } V / S}$ is made (Table II, columns 3 and 4) for the simple geometry of a stepped capillary.

Thus, an experimental value of the average 'effective' radius of $0.06 \mu \mathrm{m}$ is obtained for the $25-37 \mu \mathrm{m}$ packed bed, which is nearly two orders of magnitude smaller than $r_{\text {avg }}$ of $3 \mu \mathrm{m}$.

It is important to highlight the concept of 'effective' hydrodynamic radius, $r_{\text {eff }}$ (Table I), as it represents a uniform capillary, which shows similar flow kinetics as the porous bed. It is 
known that steady-state flow kinetics in a uniform capillary is a balance between the capillary driving force $\left(\propto 1 / r_{\text {uniform }}\right)$ and the viscous retarding force ( $\propto 1 / r_{\text {uniform }}^{2}$ ) where $r_{\text {uniform }}$ refers to the physical radius of the capillary. We notice that as the capillary radius becomes smaller, the two terms diverge, i.e., the viscous drag term increases much faster than the corresponding increase of the capillary driving force, thereby leading to decreasing flow velocities with a decrease in radius. An extremely small effective radius indicates flow kinetics limited by a large pressure drop due to periodic expansion-contraction segments during fluid flow in pores and pore necks. In order to understand the influence of such irregularities on flow rates, a nonuniform capillary model was developed.

\section{Modeling Fluid Flow in Model Porous Media: Non-Uniform Capillary}

Real flow path in a porous solid involves turtuosity and periodic changes in cross-sectional area. A sinusoidal capillary with converging-diverging sections is assumed to be representative of flow through pores and pore necks. An incompressible, laminar flow is assumed. ${ }^{6,16,17,19}$ A simple extension of Hagen-Poiseuille's law for steady-state flow is adopted for the present geometry. Equations describing the velocity of the meniscus as it rises in the capillary are derived. We further restrict the model to small heights (typically within $10 \%$ of the equilibrium height for the capillary) where the gravitational potential term can be neglected. Although the flow is not steady state and varies with time, at any given instant of time, flow can be considered to be quasi steady-state and thus Hagen-Poiseuille's law is applicable. Figure 4 shows a sinusoidal capillary with a profile defined as

$$
r=r_{\mathrm{o}}+A \sin \left(\frac{2 \pi z}{\lambda}\right)
$$

where $r_{\mathrm{o}}=$ average radius, $A=$ amplitude of variation, $z=$ length along profile, and $\lambda=$ wavelength of fluctuation.

The wavelength of variation, $\lambda$, which represents the average length between a pore and pore neck, is typically found to be equal to the average particle size for random porous media considered (based on observations of optical and scanning electron micrographs of polished sections).

Let us consider the situation when the meniscus is moving through the expanding portion of the profile. This is represented in Fig. 4 (inset) along with the coordinate system of reference.

At any instant of time $t$, fluid has risen up to a height $h$, which is the instantaneous height. The total pressure drop between the meniscus and the far-field fluid level in the reservoir can be obtained by integrating Hagen-Poiseuille's equation as

$$
\Delta P=\frac{8 \eta \dot{Q}}{\pi} \int_{h_{\text {inital }}}^{h_{\text {inst }}} \frac{\mathrm{d} z}{r^{4}(z)}
$$

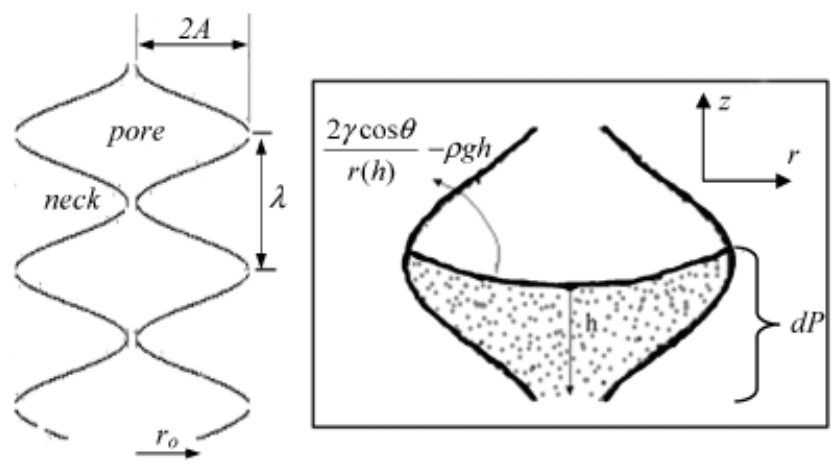

Fig. 4. Profile of sinusoidal capillary and relevant forces on the meniscus in the diverging portion. where $\dot{Q}=$ instantaneous volume flow rate, $h_{\text {initial }}=$ initial liquid height inside the profile to begin with to avoid singularity, $h_{\text {inst }}=$ instantaneous height, and $\eta=$ liquid viscosity.

The total effective pressure across the meniscus due to capillarity can be expressed as

$$
\Delta P=\frac{2 \gamma \cos \theta}{r(h)}
$$

where $\gamma=$ liquid-vapur interfacial tension, $\theta=$ contact angle (typically the static contact angle from a sessile drop experiment), and $r(h)=$ radius at height $h$.

As the capillary profile is not parallel to the $z$-axis, the 'effective,' contact angle for the meniscus leads to modification of Eq. (5) as

$$
\Delta P=\frac{2 \gamma_{l v}}{r}\left[\cos \theta-\frac{2 \sin \phi}{\{1+\sin (\theta+\phi)\}}\right]
$$

where $\tan \phi=\frac{\mathrm{d} r}{\mathrm{~d} h}=\frac{2 \pi A}{\lambda} \cos \left(\frac{2 \pi h}{\lambda}\right)$ is the instantaneous slope of the sinusoidal profile. We notice that the condition, $\Delta P=0$, can be satisfied far below the equilibrium height if $\cos \theta=\frac{2 \sin \phi}{\{1+\sin (\theta+\phi)\}}$. The instantaneous volumetric flow rate $\dot{Q}$ in Eq. (4) can be written as

$$
\dot{Q}=\pi r^{2}(h) \frac{\mathrm{d} h}{\mathrm{~d} t}
$$

Combining Eqs. (4), (5a) and (6), we have

$$
\begin{aligned}
\frac{\mathrm{d} h}{\mathrm{~d} t}= & {\left[\frac{2 \gamma}{r(h)}\left(\cos \theta-\frac{2 \sin \theta}{\{1+\sin (\theta+\varphi)\}}\right)\right] } \\
& \times \frac{1}{8 \eta r^{2}(h) \int_{h_{\text {initial }}^{h_{\text {inst }}} \frac{\mathrm{d} z}{r^{4}(z)}}}
\end{aligned}
$$

This equation was solved numerically to obtain height-time plots.

Figure 5 shows the numerical output of capillary rise kinetics for a $1.4-10.8 \mu \mathrm{m}$ sinusoidal capillary (representative of the 25 $37 \mu \mathrm{m}$ porous bed) for various $\lambda$. The height-time profile is seen to have jumps alternating with relatively flatter segments. Such features in the rise profile are found to correspond to fluid rise in the narrow segments and wide segments of the capillary, respectively. Diverging sections, where the meniscus takes a longer time to traverse, clearly control fluid flow rates.

The maximum Reynolds number $(\mathrm{Re})$ for the $1.4-10.8 \mu \mathrm{m}$ sinusoidal capillary (equivalent to the 25-37 $\mu \mathrm{m}$ packed bed) was computed to be 0.1 , indicating laminar flow. Of course, this analysis is valid at least a distance (equal to the average capillary size) away from the entrance of the capillary, because at lengths very close to the tip of the capillary, the meniscus velocity is extremely large.

Moreover, the plots shown in Fig. 5 can be fitted to a parabolic function using the static contact angle in the Washburn equation for the equivalent uniform capillary, indicating overall steady-state laminar flow behavior. The 'effective' Washburn radius for typical values of $\lambda$ between 25 and $37 \mu \mathrm{m}$ ( $\lambda$ scaling with the particle size) was calculated to be $0.04 \mu \mathrm{m}$. Surprisingly, for $\lambda>30 \mu \mathrm{m}$, the 'effective' hydrodynamic radius of $0.04 \mu \mathrm{m}$ was seen to be $\lambda$ independent. Pore tortuosity was neglected in the developed model. Incorporation of a tortuosity factor of 2 into height, $h$, would further reduce the calculated 'effective' radius by a factor of 4 (as $r_{\text {eff }} \propto h^{2}$ ), thereby leading to a value of $0.01 \mu \mathrm{m}$. Considering $90 \%$ of the volume pore size distribution, we obtain an effective pore size distribution of $2.2-10.3 \mu \mathrm{m}$ and an 'effective' radius of $0.17 \mu \mathrm{m}(0.04 \mu \mathrm{m}$ considering the tortuosity factor). These values are in good agreement with the experimentally obtained value of $0.06 \pm 0.02 \mu \mathrm{m}$ (for the $25-37 \mu \mathrm{m}$ 


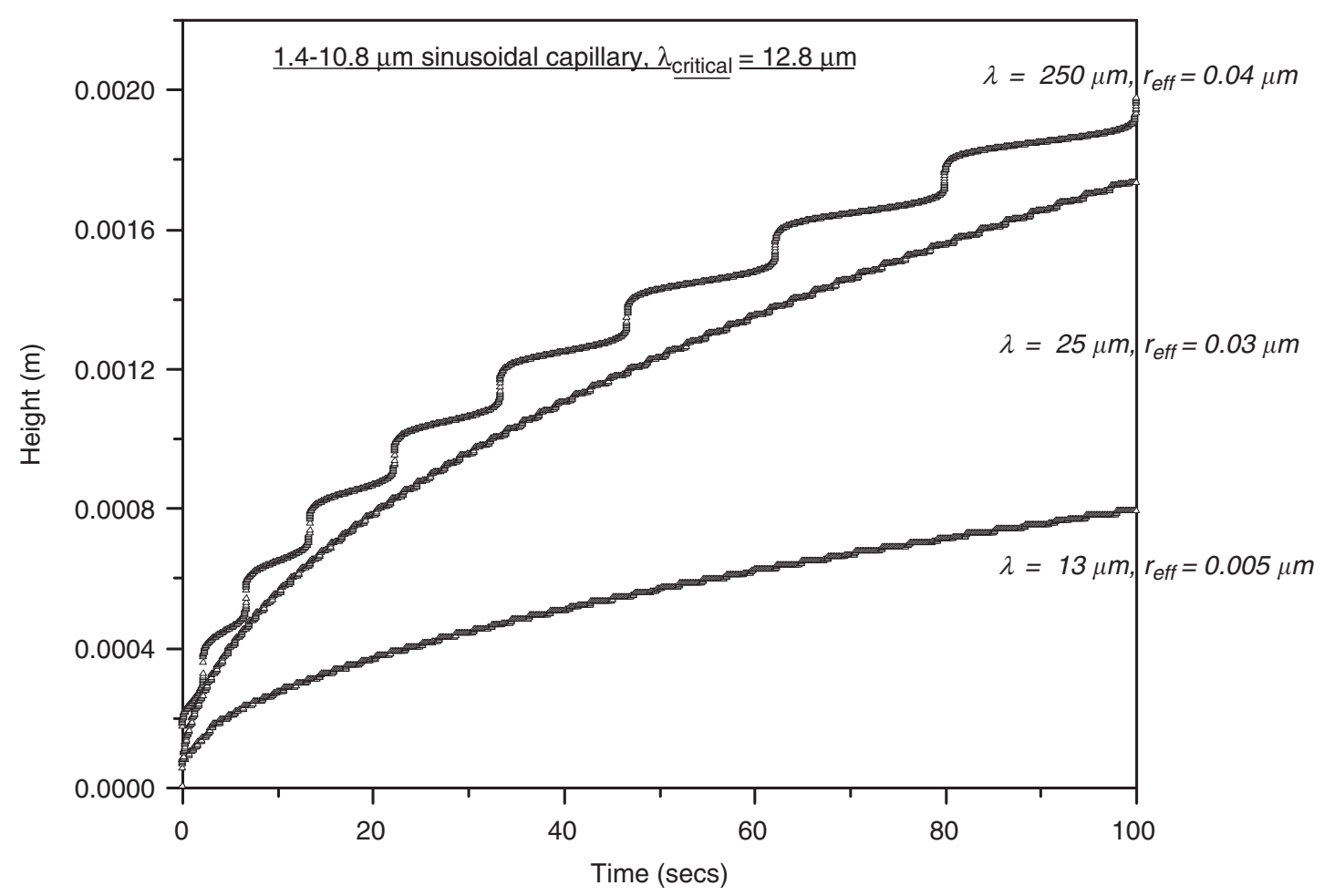

Fig. 5. Capillary rise kinetics in the 1.4-10.8 $\mu \mathrm{m}$ sinusoidal capillary as a function of $\lambda$ and the corresponding 'effective' radii obtained from parabolic Washburn fits.

bed), considering the uncertainty in determining the relevant parameters $r_{\mathrm{o}}$ and $A$ from pore size distribution.

Real porous media have a distribution of pore opening $\left(r_{\mathrm{o}}+A\right)$ and pore neck $\left(r_{\mathrm{o}}-A\right)$ sizes. $\mathrm{A} \pm 25 \%$ variation in $r_{\text {min }}$ and $r_{\max }$ results in 'effective' radii in the range $0.005-0.02 \mu \mathrm{m}$ (incorporating a factor of 2 for the tortuosity parameter). This is still within an order of magnitude of the experimentally observed effective radius of $0.06 \mu \mathrm{m}$, implying a good agreement between experiments and modeling.

The influence of the effective contact angle on the infiltration kinetics was examined for wavelengths between the critical wavelength, $\lambda_{\text {critical }}$, and the $\lambda$-independent value. For $\theta=41^{\circ}$, the limiting wall slope for spontaneous rise is $\varphi=49^{\circ}$. This condition of meniscus arrest, i.e., $\varphi=\pi / 2-\theta$, gives the equality, $\cot$ $\theta=\tan \varphi$ (from Eq. $(5 a)$ ), and the critical $\lambda$ as

$$
\lambda_{\text {critical }}=\frac{2 \pi A}{\cot \theta}
$$

A critical $\lambda$ of $12.8 \mu \mathrm{m}$ is obtained for this case, below which the meniscus is arrested and is found to be determined by the amplitude of waviness, $A$ (geometrical parameter), and wettability, $\theta$ (fluid property). A similar expression has been derived ${ }^{24}$ for demarcating the limit between wettability and repellency in a non-uniform capillary. In the extreme case, for close-packed arrangements of spheres, the critical contact angle for spontaneous infiltration has been reported to be $51^{\circ}$, above which the meniscus is arrested ${ }^{25}$ for all orientations.

At values close to $\lambda_{\text {critical }}$, infiltration was found to be further slowed down by an order of magnitude $\left(r_{\text {eff }}=0.004 \mu \mathrm{m}\right)$ compared with the $\lambda$-independent value $(\sim 0.04 \mu \mathrm{m})$. This implies that the 'effective' hydrodynamic radius of a non-uniform capillary is dependent on both the geometry $\left(r_{\mathrm{o}}\right.$ and $\left.A\right)$ as well as the 'effective' local contact angle. Thus, the effect of local contact angle cannot be ignored in a particle packing, more so when the 'effective' contact angle $(\theta+\varphi)$ is $>80^{\circ}$.

In general, $\lambda$ scales with the average particle size, but there is a statistical distribution of $\lambda$ in the porous medium. The packing inhomogeneity in the porous bed leads to local regions wherein $\lambda<\lambda_{\text {critical }}$, and such regions are not directly filled with the advancing meniscus but have to wait for the fluid to come around and fill these regions due to the $3 \mathrm{D}$ interconnectivity. In other regions of the packed bed, there is a positive driving force for capillary flow, with regions having $\lambda$ close to $\lambda_{\text {critical }}$ filling slower than regions with $\lambda>\lambda_{\text {critical. }}$ Fluid flow prefers the path of least resistance (i.e., preferential flow channels) within the porous medium and the volumetric flow rate is controlled by such channels. The macroscopic kinetics (as measured in the experiments) can thus be adequately represented by such nonuniform preferential flow paths, thereby leading to good agreement between the observed values and the modeled values. It might be noted that in the present case $(25-37 \mu \mathrm{m}$ bed), the experimentally observed $\lambda(\approx 25 \mu \mathrm{m})>\lambda_{\text {critical }}$ where the flow becomes insensitive to wavelength (Fig. 5).

\section{Origin of 'Effective' Dimension of Non-Uniform Capillary}

Experimental values for $25-37 \mu \mathrm{m}$ porous bed give us an 'effective' radius that is two orders of magnitude smaller than the smallest physical dimensions in the porous bed. Similar results were obtained from the modeled 'effective' radius for the 25-37 $\mu \mathrm{m}$ bed in the preceding section. It is imperative to interpret this extremely small 'unphysical' dimension (smaller than the smallest actual pore size in the bed), which dictates kinetics of capillary rise inside a porous bed. To explain the physics of the flow, we consider a simpler structural model of a stepped capillary (Fig. 6) with profile parameters $r_{\min }=$ smaller segment radius, $r_{\max }=$ large segment radius, and $l=$ segment length, which is same for both segments.

Such a capillary has the essential feature of the porous medium, viz., periodic expansions and contractions, without the effect of local contact angle due to the vertical wall profile of the stepped capillary. This leads to a $\lambda$-independent infiltration kinetics for the stepped capillary and gives an opportunity to investigate the geometry-dependent $\left(r_{\mathrm{o}}, A\right)$ and $\lambda$-insensitive effect of the converging-diverging profile in influencing the capillary flow kinetics for the sinusoidal capillary (representative of the $25-37 \mu \mathrm{m}$ particle size-packed bed). For even finer preforms 


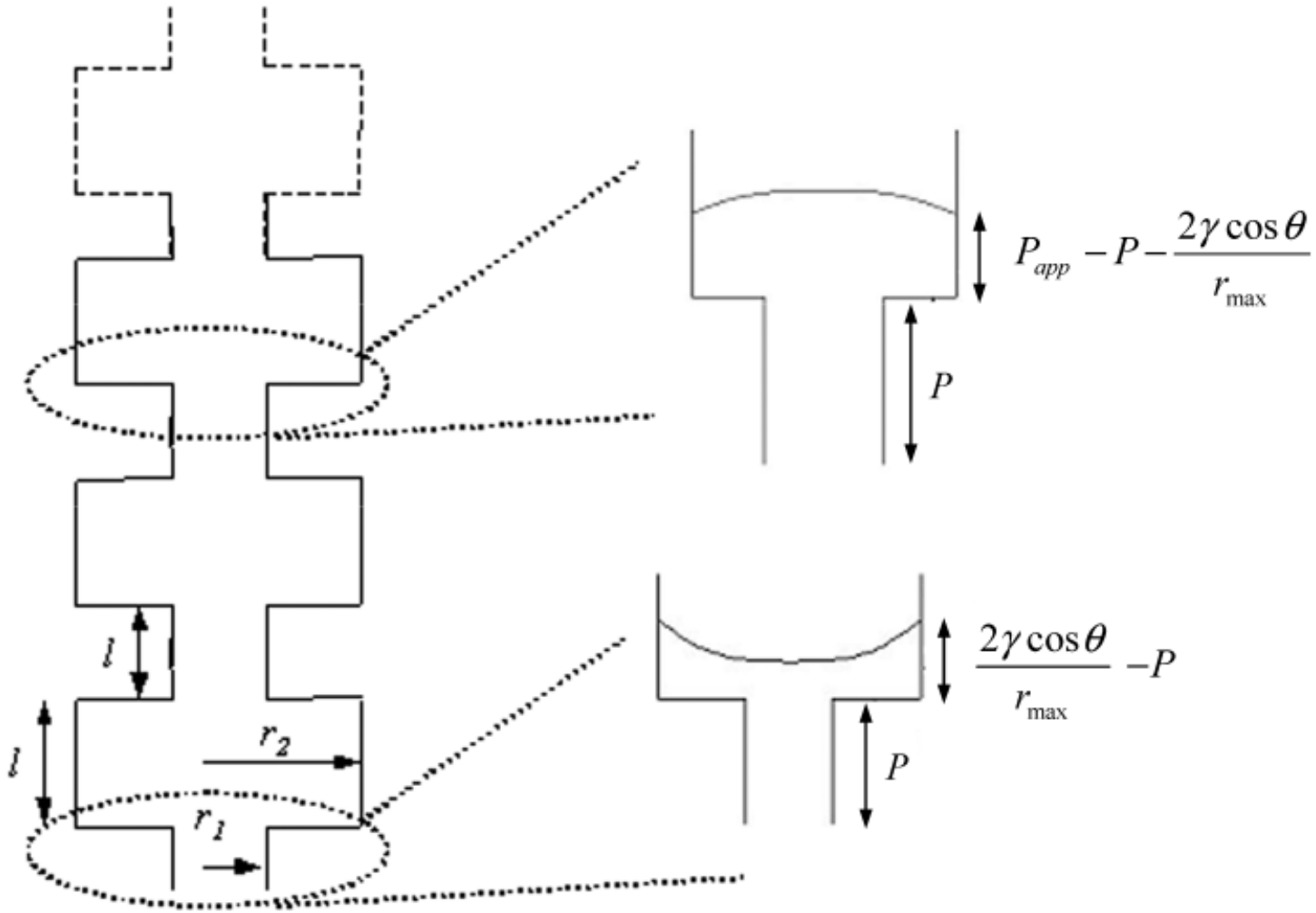

Fig. 6. Stepped capillary with alternate converging-diverging sections. Corresponding situations for wetting (bottom right) and non-wetting (top right) situation are shown.

where the average wavelength may be below the limit for arrest, this analysis developed subsequently would still apply to locally inhomogeneous regions of longer wavelength that could allow some transport.

Also shown in Fig. 6 is an enlarged region of one such diverging segment. Marked in the figure is the effective driving force for fluid flow in the two segments as will be discussed subsequently. Flow velocity in the broader segment was derived assuming a laminar, quasi steady-state flow.

Equating the flow rates in both segments, we have

$$
\dot{Q}_{1}=\dot{Q}_{2}
$$

and expressing them using Hagen-Poiseuille's formulation, we have

$$
\pi r_{2}^{2} v=\frac{\pi r_{1}^{4}}{8 \eta}\left(\frac{P}{h}\right)
$$

\section{(1) Wetting Situation (Pressureless)}

Consider the wetting case with contact angle $\theta<90^{\circ}$. The driving pressure, $P$, in the narrow segment can be expressed as

$$
P=\left(\frac{l}{h \alpha^{4}+l}\right)\left(\frac{2 \gamma \cos \theta}{r_{\max }}\right)
$$

where $\alpha=\left(r_{\min } / r_{\max }\right)$ is the radius ratio of the two segments and $h=$ instantaneous height of meniscus in segment 2 .

Substituting for $P$ from above, after approximation and rearranging Eq. (9), we obtain

$$
v=\frac{\gamma \cos \theta}{4 \eta h}\left(\frac{r_{\min }^{4}}{r_{\max }^{3}}\right) .
$$

When this is compared with the flow velocity for a fluid moving under the action of a capillary driving force through a uni- form radius ' $r$ ' (as per Eq. (1)), we have

$$
r_{\mathrm{eff}}=\frac{r_{\min }^{4}}{r_{\max }^{3}} .
$$

Such a representation correlates the geometrical parameters of a stepped capillary (representative of a porous medium) with an equivalent uniform capillary. The 'effective' uniform hydrodynamic capillary radius, $r_{\text {eff }}$ (Eq. (12)), is significantly different from the mean average radius $r_{\text {avg }}$ or 'equivalent hydraulic' radius $r_{\mathrm{avg}, V / S}$ of the stepped capillary as indicated in Table II. Also shown in Table II are the 'effective radii' derived from parabolic fits to the simulated plots for different stepped capillaries. The simulated plots were numerically generated using Eq. (7) previously derived for flow velocity. Trends in the simulated values (column 6, Table II) and values according to the Eq. (12) (column 5, Table II) are in good agreement.

Previously, a similar expression for the 'effective' radius in a non-uniform capillary under wetting conditions has been reported $^{19}$ and good agreement has been found for radii ratio $\left(r_{\max } / r_{\min }\right)$ up to 10:1. Although such an expression gives values matching the experimental results, they are physically unsound as the area ratio term is neglected. The physical interpretation of the expression in Eq. (12) is presented below

$$
\begin{aligned}
& \frac{r_{\min }^{4}}{r_{\max }^{3}}=\left(\frac{1 / r_{\max }}{1 / r_{\min }^{2}}\right) \times\left(\frac{r_{\min }^{2}}{r_{\max }^{2}}\right) \\
& =(\text { ratio of capillary driving force to viscous retarding force }) \\
& \quad \times \text { (ratio of areas) }
\end{aligned}
$$

Thus, the expression used in the present study is more meaningful, physically. 


\section{(2) Non-Wetting Situation (Pressure-Assisted Infiltration)}

In a non-wetting situation, liquid has to be forced with external pressure (Fig. 6) as in squeeze casting. Similar to the pressureless situation, it can be shown that the meniscus velocity in the broader rate-controlling segment is

$$
v=\frac{P_{\mathrm{app}}}{8 \eta h}\left(\frac{r_{\min }^{4}}{r_{\max }^{2}}\right)
$$

Compared with the uniform capillary flow (Eq. (1)), we derive the 'effective' capillary radius to be

$$
r_{\text {eff }}=\frac{r_{\text {min }}^{2}}{r_{\max }}
$$

Of course, this equality is strictly valid when the applied pressure $P_{\text {app }}$ is much larger than the threshold pressure $P_{\text {th }}$ for infiltration. The difference between the two situations $\left(r_{\text {eff }}\right.$ from Eq. (12) vs. Eq. (15)) derives from the fact that the driving pressure in the broader segment is independent of the segment size for pressure infiltration.

\section{Conclusions}

In this work, a non-uniform capillary model has been developed to explain the capillary rise kinetics inside porous $\mathrm{Al}_{2} \mathrm{O}_{3}$ compacts from the standpoint of a purely physical process. The effective hydrodynamic radius of the porous medium ' $r$ eff' has been derived and correlated both to the geometry of the porous medium, viz., minimum pore size and maximum pore size as well as 'effective' local contact angle. The 'effective' radius was found to be one to two orders of magnitude smaller than the average physical dimension derived from pore size distribution. This leads to anomalously slow capillary rise rates. Such an 'unphysical' length scale was rationalized based on an interplay between capillary driving force and viscous retarding force, coupled with the inherent converging-diverging geometry of the porous medium, where additional retardation effects associated with a large 'effective' local contact angle can be neglected.

\section{Acknowledgments}

We would like to acknowledge B. P. Saha, Dr. U. Hareesh and others at ARCI, Hyderabad, India, for their help in preparing the slip-cast samples and conducting mercury porosimetry. We thank Prof. R. K. Bordia (University of Washington, Seattle) and Prof. V. A. Ravi (California State Polytechnic University) for useful suggestions.

\section{References}

${ }^{1}$ M. S. Newkirk, A. W. Urquhart, H. R. Zwicker, and E. E Breval, "Formation of Lanxide Ceramic Composite Materials," J. Mater. Res., 1 [1] 81-9 (1986).

${ }^{2}$ M. K. Aghajanian, J. T. Burke, D. R. White, and A. S. Nagelberg, "New Infiltration Process for the Fabrication of Metal Matrix Composites," SAMPE Q, 20 [4] 43-6 (1989).

${ }^{3}$ C. W. Forrest, P. Kennedy, and J. V. Shennan, Special Ceramics 5. Proceedings of British Ceramic Research Association, p. 99, Stoke-on-Trent, U.K., 1972.

${ }^{4}$ B. S. Rao and V. Jayaram, "Pressureless Infiltration of Al-Mg Based Alloys into $\mathrm{Al}_{2} \mathrm{O}_{3}$ Preforms: Mechanisms and Phenomenology," Acta. Mater., 49 [13] 2373-85 (2001).

${ }^{5}$ D. Y. Lee, D. J. Kim, B. Y. Kim, and Y. S. Song, "Effect of Alumina Particle Size and Distribution on Infiltration Rate and Fracture Toughness of AluminaGlass Composites Prepared by Melt Infiltration," Mat. Sci. Eng. A, 341 [1-2] 98-105 (2003).

${ }^{6} \mathrm{E}$. O. Einset, "Capillary Infiltration Rates into Porous Media with Applications to Silcomp Processing," J. Am. Ceram. Soc., 79 [2] 333-8 (1996).

${ }^{7}$ P. Yi, S. Y. Xiao, and J. L. Baptista, "Kinetics of Cobalt Silicide Infiltration into Silicon Carbide Preforms," J. Am. Ceram. Soc., 82 [12] 3459-65 (1999).

${ }^{8}$ C. Toy and W. D. Scott, "Ceramic-Metal Composite Produced by Melt Infiltration," J. Am. Ceram. Soc., 73 [1] 97-101 (1990).

${ }^{9}$ D. Muscat, R. L. Harris, and R. A. L. Drew, "The Effect of Pore Size on the Infiltration Kinetics of Aluminum in Titanium Carbide Preforms," Acta Metal. Mater., 42 [12] 4155-63 (1994).

${ }^{10}$ A. Mortensen, B. Drevet, and N. Eustathopoulos, "Kinetics of DiffusionLimited Spreading of Sessile Drops in Reactive Wetting," Scripta Mater., 36 [6] 645-51 (1997).

${ }^{11}$ O. Dezellus, F. Hodaj, and H. Eustathopoulos, "Progress in Modeling of Chemical-Reaction Limited Wetting," J. Eur. Ceram. Soc., 23 [15] 2797-803 (2003).

${ }^{12}$ D. Muscat and R. A. L. Drew, "Modeling the Infiltration Kinetics of Molten Aluminum into Porous Titanium Carbide," Metall. Trans. A, 25A [11] 2357-70 (1994).

${ }^{13}$ V. Bougiouri, R. Voytovych, N. Rojo-Calderon, J. Narciso, and N. Eustathopoulos, "The Role of Chemical Reaction in the Infiltration of Porous Carbon by NiSi Alloys," Scripta Mater., 54 [11] 1875-8 (2006).

${ }^{14}$ P. C. Carman, "Fluid Flow through Granular Beds," Trans. Inst. Chem. Eng., 15, 150-66 (1937)

${ }^{15}$ E. W. Washburn, "The Dynamics of Capillary Flow," Phys. Rev., 17 [3] 273-8 (1921)

${ }^{16}$ G. P. Martins, D. L. Olson, and G. R. Edwards, "Modeling of Infiltration Kinetics for Liquid Metal Processing of Composites," Metall. Trans. B, 19B [1] 95-101 (1988).

${ }^{17}$ F. A. L. Dullien, M. S. El-Sayed, and V. K. Batra, "Rate of Capillary Rise in Porous Media with Non-uniform Pores," J. Coll. Int. Sci., 60 [3] 497-506 (1977).

${ }^{18}$ R. Sharma and D. S. Ross, "Kinetics of Liquid Penetration into Periodically Constricted Capillaries," J. Chem. Soc. Far. Trans., 87 [4] 619-24 (1991).

${ }^{19}$ T. L. Staples and D. G. Shaffer, "Wicking Flow in Irregular Capillaries," Coll. Surf. A, 204 [1-3] 239-50 (2002).

${ }^{20}$ I. Fatt, "The Network Model of Porous Media I, II and III," Petrol. Trans. AIME, 207, 144-77 (1956).

${ }^{21}$ F. A. L. Dullien, "New Network Permeability Model of Porous Media," AIChE J., 21 [2] 299-307 (1975).

${ }^{22}$ A. Siebold, A. Walliser, M. Nardin, M. Oppliger, and J. Schultz, "Capillary Rise for Thermodynamic Characterization of Solid Particle Surface," J. Coll. Int. Sci., 186 [1] 60-70 (1997).

${ }^{23}$ G. E. P. Elliott and A. C. Riddiford, "Dynamic Contact Angles I. The Effect of Impressed Motion," J. Coll. Int. Sci., 23 [3] 389-98 (1967).

${ }^{24}$ Henryk Czachor, "Modeling the Effect of Pore Structure and Wetting Angles on Capillary Rise in Soils having Different Wettabilites," J. Hydrol., 328 [3/4] 604-13 (2006).

${ }^{25}$ K. P. Trumble, "Spontaneous Infiltration of Non-Cylindrical Porosity: Close-Packed Spheres," Acta. Mater., 46 [7] 2363-7 (1998). 